\title{
Versuche einer Visualisierung: Deutsch-Australische Bilder aus dem Ersten Weltkrieg in Westaustralien
}

\section{Historischer Bezug}

Frank. Franz. She [his widow Hannah Roennfeldt] recalls the gentle man whose life was turned upside down so many times [...]. The first reverse saw him ripped from his life of privilege in Vienna as a boy of sixteen, as his father's gambling debts drove them all the way to relatives in Kalgoorlie, a place so remote from Austria that even the most ardent creditor would give up the chase. From luxury to austerity, the son taking on the trade of baker in the shop run by his uncle and aunt, who since their arrival years before had changed from Fritz and Mitzie to Clive and Millie. It was important to blend in, they said. His mother understood this, but his father, with the pride and stubbornness that had triggered his financial ruin, resisted adaptation, and within a year had thrown himself under a train bound for Perth, leaving Frank as head of the household. Months later, war brought internment as an enemy alien - first on Rottnest Island, then over East - for this boy who was now not simply uprooted and bereaved, but despised, for things done far away and beyond his control. (Stedman 157)

Diese kleine Episode in M.L. Stedmans Bestseller The Light Between Oceans (2012), einem Roman, der in Stedmans Heimatstaat Westaustralien in der Zeit um den Ersten Weltkrieg angesiedelt ist, wirft das Schlaglicht in wenigen Sätzen auf einen gehassten "Hun", den die kleine Gemeinde aus ihren Reihen vertreibt. In Stedmans Roman taucht er nur noch als Leiche auf, doch die Einblicke, die andere Charaktere auf seine Biographie zulassen, insbesondere seine Internierung auf Rottnest Island, machen neugierig auf die tragische und wahre Geschichte hinter seinem Schicksal.

Die Internierung der deutschen Zivilbevölkerung im Ersten Weltkrieg ist kaum mehr als eine Fußnote in der Geschichtsschreibung Australiens. Ausnahmen sind Helmi et al. (2011), Simons (1999) und Fischer (1989), wobei der Westen des Kontinents meist nur am Rande behandelt wird. Im Folgenden soll erstmals die Situation in Westaustralien anhand von authentischen "Bildern" zumindest annähernd erfasst werden. 
Im August 1914, wenige Stunden nach Ausbruch des Ersten Weltkrieges, wurde Westaustralien zum Kriegsschauplatz im Indisch-Pazifischen Raum. Ein deutsches Handelsschiff wurde beschossen, seine deutsche Mannschaft festgenommen, das Schiff beschlagnahmt und die Internierung der Besatzung vorbereitet. Auf diese Menschen warteten Monate der Unsicherheit und Jahre der Internierung - ein Leben im Schwebezustand. In diesem Aufsatz möchte ich einige der dramatischen Ereignisse näher beleuchten, die von drei Seemännern visuell festgehalten wurden: von einem Hobby-Fotografen, einem Amateur-Maler und einem Laien-Kartografen. Ihre Zeitzeugnisse sind die einzigen Visualisierungen, die uns diese Geschichte heute noch nahebringen können.

\section{Der Ausbruch des Krieges}

Noch kurz vor der Kriegserklärung lag die Stolberg, ein neu gebautes Schiff aus Hamburg, im Hafen von Fremantle, verließ jedoch alarmiert durch Gerüchte, der Kriegsausbruch stehe unmittelbar bevor, in der Nacht vom vierten auf den fünften August hastig die australischen Gewässer (Minute Paper). Anstatt wie geplant nach Adelaide weiterzufahren, machte sich die Stolberg auf den Weg nach Java, um dort Schutz zu suchen. Sie fuhr also Richtung Nord/Nordwesten. Deshalb kreuzte sie unterwegs auch nicht den Pfad eines anderen deutschen Schiffes, das auf Fremantle zuhielt: die Greifswald.

An Bord der SS Greifswald war Karl Lehmann, der 1886 als einer von sieben Söhnen einer wohlhabenden deutsch-japanischen Familie in Tokio geboren wurde. Sein Vater Rudolph Lehmann und sein Onkel Carl, die aus Oldenburg stammten, waren in den 70er Jahren des 19. Jahrhunderts unter den ersten Schiffsbauern gewesen, die nach Japan gingen. Rudolph hatte dort ein fünfzehnjähriges japanisches Mädchen kennengelernt, mit der er insgesamt acht Kinder hatte. Die Söhne wurden für ihre Schulausbildung nach Deutschland geschickt. Karl Lehmann absolvierte seinen Militärdienst in Deutschland, heiratete eine deutsche Frau und visierte eine Karriere auf hoher See an. Aufgrund privater Schicksalsschläge nahmen seine Pläne jedoch einen anderen Verlauf (1911 starb sein älterer Bruder, 1913 wurde seine erste Ehe geschieden und 1914 verstarb sein Vater in Tokio). 
Am Vorabend des Ersten Weltkriegs befand sich Lehmann als dritter Offizier auf der Greifswald auf dem Weg nach Australien und hielt die wichtigsten Stationen der Reise mit einer Kamera fest: beispielsweise den Hafen von Antwerpen, den Hafen von Genua und die Anfahrt auf Australien. In den frühen Morgenstunden des 6 . August lief die Greifswald im Hafen von Fremantle ein. Eigentliches Ziel war Adelaide. Der Kapitän wurde

...vor Rottnest von einem Untersuchungsdampfer angesprochen, der den Kapitän anhielt, wie üblich vor dem Hafen zu ankern. Der Kapitän der Greifswald kam den Befehlen nichtsahnend nach. Kurz nach Tagesanbruch lichtete er den Anker und fuhr in den Hafen ein, während die Mannschaft und Offiziere damit beschäftigt waren, die Luken zu öffnen, damit die Ladung gelöscht werden konnte. Nachdem das Schiff an der Mole festgemacht hatte, kam der örtliche Handelsvertreter an Bord und informierte den ahnungslosen Kapitän, dass der Krieg begonnen hatte. Dieser war völlig verblüfft und brach in Tränen aus, als er die Neuigkeit hörte. ("The censors" 15)

Der Verlust ihrer Freiheit traf Besatzung und Kapitän vollkommen unvorbereitet. Genauso wenig waren allerdings die örtlichen Behörden darauf vorbereitet, das Schiff in Besitz zu nehmen. Die Soldaten, die in aller Eile den Befehl erhalten hatten, die Besatzung gefangen zu nehmen, waren flugs eingezogene Reservisten, denn Australien hatte zu dieser Zeit keine Berufsarmee. Einige dieser Rekruten hatte man schnell in eine Uniform gesteckt, mit einem Gewehr ausgerüstet und an die Gangway gestellt. Sie sollten Wache halten und verhindern, dass unbefugte Personen das Schiff betraten oder jemand es verließ. Zu Anfang wurden die 46 Besatzungsmitglieder nämlich auf ihrem Schiff festgehalten. Da Lehmann nicht viel zu tun hatte, experimentierte er mit seiner Kamera. Auf diese Weise entstanden in den folgenden Monaten über 300 Fotos in Westaustralien.

Obwohl die Offiziere und der Kapitän selbst Reservisten waren und ihren Dienst in der deutschen Armee abgeleistet hatten, durften sie sich im Gegensatz zum Rest der Besatzung nach einer Weile relativ frei bewegen. Sie wurden als Ehrenmänner behandelt, und der Ton zwischen den australischen Behörden und dieser Gruppe der Gefangenen war freundlich. Man hatte ihr Wohlergehen und ihre seelische Gesundheit im Sinn, als man innen erlaubte, sich in Fremantle und Umgebung umzusehen. Auf diese Weise konnte Lehmann viele Sehenswürdigkeiten der Hafenstadt fotografieren. Die Offiziere aßen auch regelmäßig mit ihren australischen Kollegen in deren Militärzel- 
ten im Fremantle Park zu Mittag. Lehmann muss sich bewusst gewesen sein, dass die Anwesenheit eines enemy aliens unter Australiern jederzeit feindselige Reaktionen hervorrufen konnte und dass es untersagt war, als Angehöriger einer Feindnation mit seinem Fotoapparat Bilder von Militärzelten und dem Hafen zu machen. Wohl deshalb schoss er ein Foto des Militärlagers im Fremantle Park aus der Hüfte. Auf keinem seiner anderen Fotos ist es Lehmann, der sonst einen ausgeprägten Sinn für Gestaltung, Komposition und Beleuchtung besaß, unterlaufen, dass man seinen eigenen Schatten auf dem Bild sehen kann oder dass sein Motiv nicht mittig platziert ist, so wie bei diesem Bild:

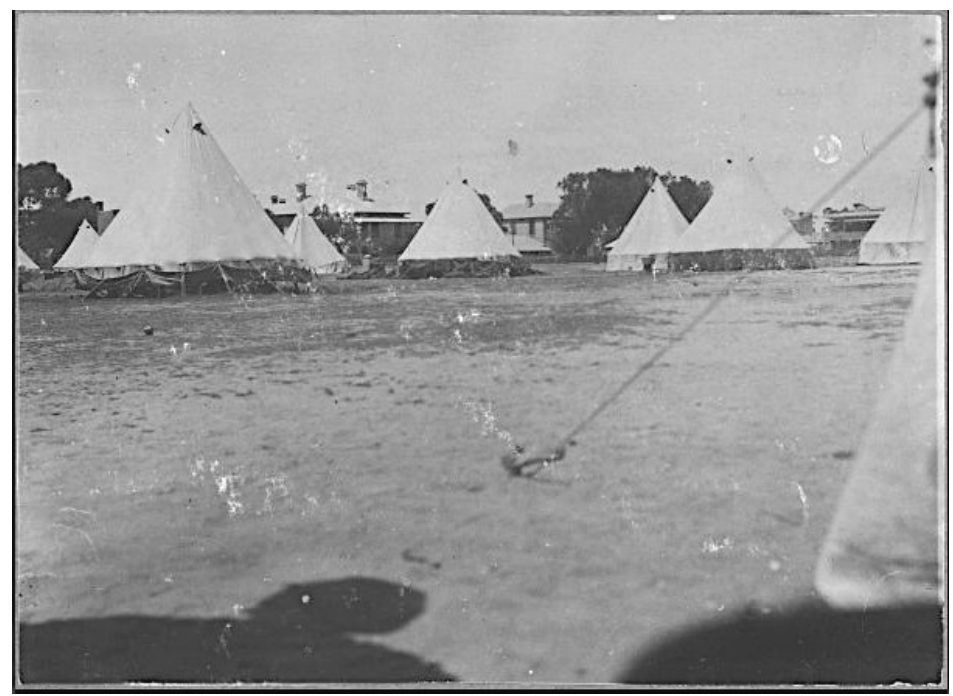

Karl Lehmann, Militärlager in Fremantle, Westaustralien, 1914, National Library of Australia, Bib ID 4864920.

\section{Die Registrierung und Festsetzung der enemy aliens}

Am 10. August 1914 war eine Regierungserklärung abgegeben worden, die von allen deutschen Staatsbürgern in Australien verlangte, sich bei der Polizei zu melden. Drei Tage später erließ der Generalgouverneur Sir Ronald Craufurd Munro Ferguson eine weitere Verlautbarung, die alle im Commonwealth lebenden Untertanen des Kaisers von Österreich (und gleichzeitigen Königs von Ungarn) auf- 
forderte, sich gleichermaßen wie die deutschen enemy aliens zur nächsten Polizeidienststelle zu begeben und dem diensthabenden Beamten Namen, Wohnort und Beschäftigung bekanntzugeben sowie den Behörden jedwede Änderungen der Personalien mitzuteilen (Commonwealth of Australia Gazette 56). In Fremantles Polizeistation und Kaserne war daraufhin viel los: Geheimdienstoffiziere führten Gespräche mit enemy aliens und kalkulierten den Grad der Bedrohung, der von ihnen ausging. Der Zugang zum Kai wurde eingeschränkt, ebenso der Besitz von Waffen oder Fotoapparaten.

Lehmann kann nicht entgangen sein, dass immer mehr Deutsche und Österreicher nach ihrer Registrierung - unabhängig davon, ob sie erst kürzlich im Land angekommen waren oder schon lange dort wohnten - als enemy aliens festgenommen und an verschiedenen Orten innerhalb Fremantles interniert wurden: z.B. im Esplanade Hotel und dem ihm vorgelagerten Park, im nahe gelegenen Fremantle Park und in den Kasernen der Artillerie. Die Behörden waren aber darauf aus, die Gefangenen an Orte zu verlegen, die nicht von militärischer Bedeutung waren. Trotz der Pressezensur, die es verbot, über die Internierung der enemy aliens zu berichten (Scott 66), fanden hier und da Bruchstücke dieser Nachricht ihren Weg in die Zeitungen. Daraus geht hervor, dass bereits am 14. August bekannt war, dass mehrere Deutsche "auf der bekannten Ferieninsel Rottnest" festgesetzt worden waren ("Timber topics" 4). Die Insel war wenige Tage nach Ausbruch des Krieges unter Militärverwaltung gestellt und als Internierungslager hergerichtet worden. Nun wurden Wachen mobilisiert, Vorräte zusammengetragen und alles Notwendige mit kleinen Passagierdampfern auf die neun Meilen vom Festland entfernte Insel transportiert.

Sonst war der Hafen wenig geschäftig und die Hafenanlage nahezu menschenleer. Die örtliche Wochenzeitung der Labor Party, The Westralian Worker, berichtete: "In der letzten Woche war es am Kai außergewöhnlich ruhig. Kein Ozeandampfer wurde beladen und das Ausbleiben der deutschen Dampfschiffe ist deutlich zu spüren." ("Notes from the sea" 10). 


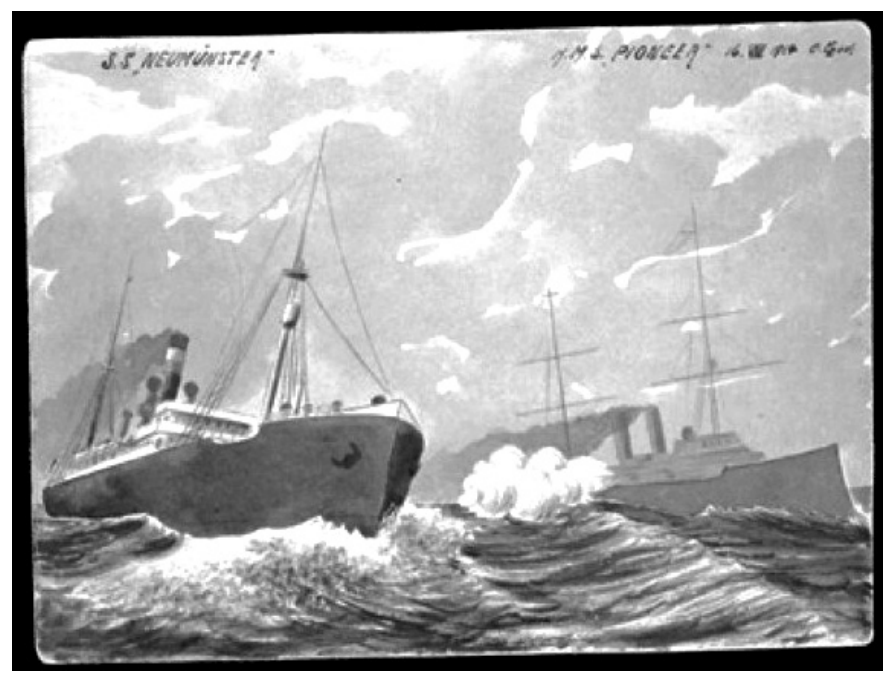

Fotografie einer Zeichnung von Erich Czech 1914, State Library of Western Australia, Picture 280261PD.

An der gesamten Westküste war es friedlich, bis völlig unerwartet ein weiteres deutsches Schiff auftauchte, die Neumünster. An Bord dieses Schiffes war Erich Czech, damals 31 Jahre alt, ein Vollmatrose. Im Gegensatz zu Lehmann hatte Czech zwar keine Kamera dabei, dafür war er mit Leinwand und Pinsel ausgerüstet. Die folgende Begegnung hat er in Wasserfarben festgehalten.

Am frühen Morgen des 16. August entdeckte die H.M.A.S. Pioneer etwa 9 Meilen vor Rottnest die Lichter eines sich nähernden Schiffes. Das Kriegsschiff fuhr ohne Licht auf das unbekannte Schiff zu. Erst als es sich bis auf eine Dreiviertelmeile genähert hatte, schaltete die Pioneer die Schiffsbeleuchtung an, umrundete das abgefangene Boot von Steuerbord nach Backbord und sandte eine Botschaft, in der nach dem Namen des Schiffes gefragt wurde. Außerdem wurde ein Schuss über Bug gefeuert, verbunden mit der Aufforderung anzuhalten. Als keine Reaktion erfolgte, wurde ein zweites Mal gefeuert. Erst darauf hielt das Dampfschiff an. (Unmack quoted in "Prize court" 7)

Da die Neumünster nicht mit einem Funkradio ausgestattet war, hatte der Kapitän Karl Hermann noch nichts von der Kriegserklärung gehört und mutmaßte, dass die Warnschüsse etwas damit zu tun haben könnten, dass er sich bei der Anfahrt auf Fremantle gefährlichen Riffen und Felsen angenähert habe. Sein Schiff war voll beladen mit 
Waren aus Antwerpen (wovon 600 Tonnen in Fremantle gelöscht werden sollten) und Hermann wollte seine Jungfernfahrt als Kapitän unbedingt ohne Zwischenfall hinter sich bringen ("Captured off Fremantle" 14).

Auch vor Rottnest Island war es schwierig, den Kontakt zu dem Schiff herzustellen. In Ermangelung anderer Möglichkeiten wurde ein Megaphon benutzt, um den Kapitän darüber in Kenntnis zu setzen, dass ein Boot zu ihm hinübergeschickt werde ("Prize court" 7). Als sich dies aufgrund der rauen See als unmöglich erwies, signalisierte die Pioneer: "Maschinen stoppen. Sie werden eskortiert" ("Rottnest Island" 2). Zu diesem Zeitpunkt - es war ein dunkler, stürmischer Wintermorgen - konnten der Kapitän und seine Mannschaft unmöglich erkennen, dass die Pioneer ein Kriegsschiff war. Schließlich setzte der Lotse Alexander Williamson aus Fremantle über, der das Schiff in den Hafen steuerte und dort vertäute. Daraufhin wurde das Schiff offiziell beschlagnahmt und weitere bewaffnete Wachen abgestellt. Erst dann war die Nachricht vom Kriegsausbruch bis zur Besatzung durchgedrungen. Der letzte Befehl, nachdem das Schiff am Viktoriakai festgemacht worden war, lautete: "Alle an Bord bleiben!" Die 42-köpfige Besatzung der Neumünster, unter ihr der Seefahrer Erich Czech, wurde auf dem Frachtdampfer festgehalten, bis sie am 21. August 1914 mit einem anderen Boot nach Rottnest Island gebracht wurde. Czech war ein geschickter Maler und Musiker, und für beide Hobbys sollte er in den kommenden Jahren noch viel Zeit haben. Sowohl er als auch Karl Lehmann gehörten zu den Deutschen, die erst im Hafen festsaßen, bevor sie auf Rottnest Island interniert wurden, wo sie Ende August als Gefangene Nr. 190 und Nr. 33 registriert wurden.

\section{Die Internierung auf Rottnest Island}

Bereits am 13. August, kaum eine Woche nach Ausbruch des Krieges, entschieden sich die Militärbehörden für Rottnest Island als Internierungslager, vor allem weil es dort "vielfältige Unterkunftsmöglichkeiten" gab ("Western Australia" 14). Die Schiffsoffiziere wurden im State Hostel untergebracht, einer ehemaligen Erziehungsanstalt für Jungen, und auf dem Gelände des früheren Aboriginal-Gefängnisses (The Quod), das erst kurz zuvor in eine Touristenunterkunft umgebaut worden war. 


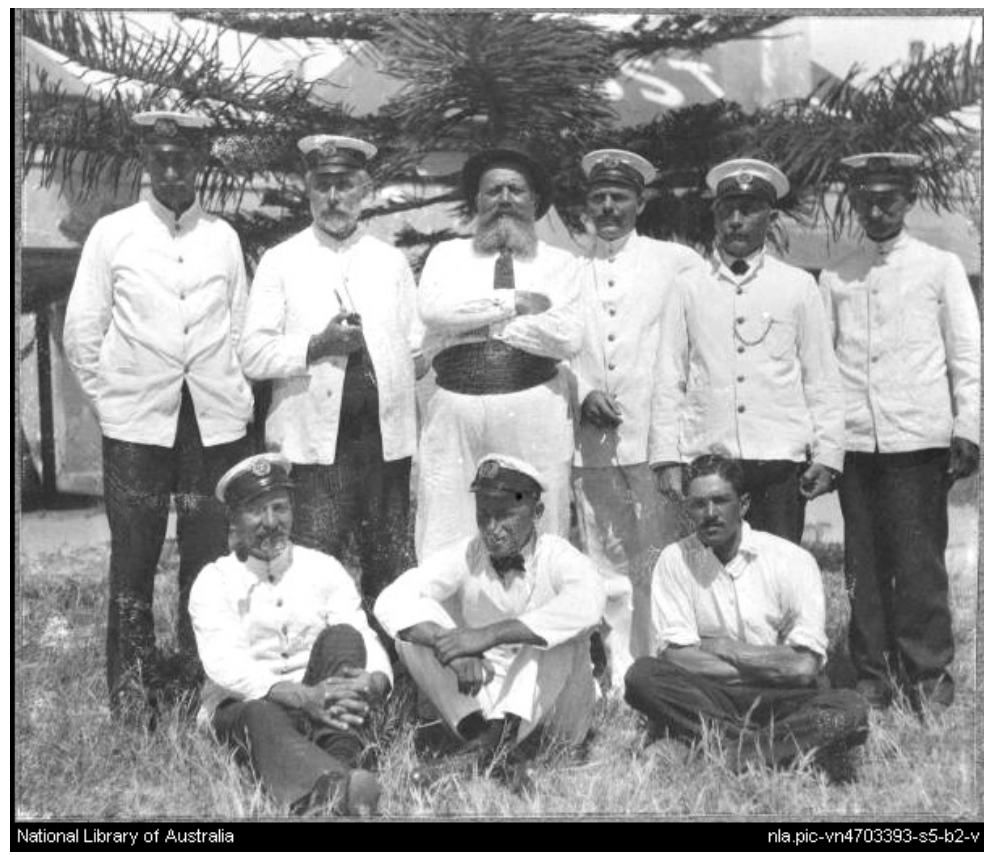

Karl Lehmann, Offiziere der SS Greifswald in Fremantle, Westaustralien, 1914. National Library of Australia, Bib ID 4703393

(Karl Lehmann sitzt in der ersten Reihe ganz rechts).

Die Mitglieder der Schiffsbesatzungen und die Zivilisten kamen in quadratischen Zelthütten unter, die mit Holzrahmen verstärkt und mit einem Holzfußboden und einem hölzernen Umlauf ausgestattet worden waren (Mennicken-Coley 55).

Czech hat auf diesem Aquarell das Zelt Nr. 31, in dem er untergebracht war, gezeichnet. Es befand sich direkt neben dem Lagereingang. Alle diese Zeltunterkünfte waren "von den Deutschen belegt, da sie die ersten waren, die eingesperrt wurden" (Splivalo 67). Tatsächlich waren die ersten 300 Internierten mehrheitlich Matrosen. Sie alle entstammten deutschen Mannschaften, denn noch am 28. August 1914 hatte ein ahnungsloser Kapitän sein Schiff, die Thüringen, in den Hafen von Fremantle gesteuert, so dass drei große deutsche Handelsschiffe, die Greifswald, die Neumünster und die Thüringen in Fremantle festlagen. 


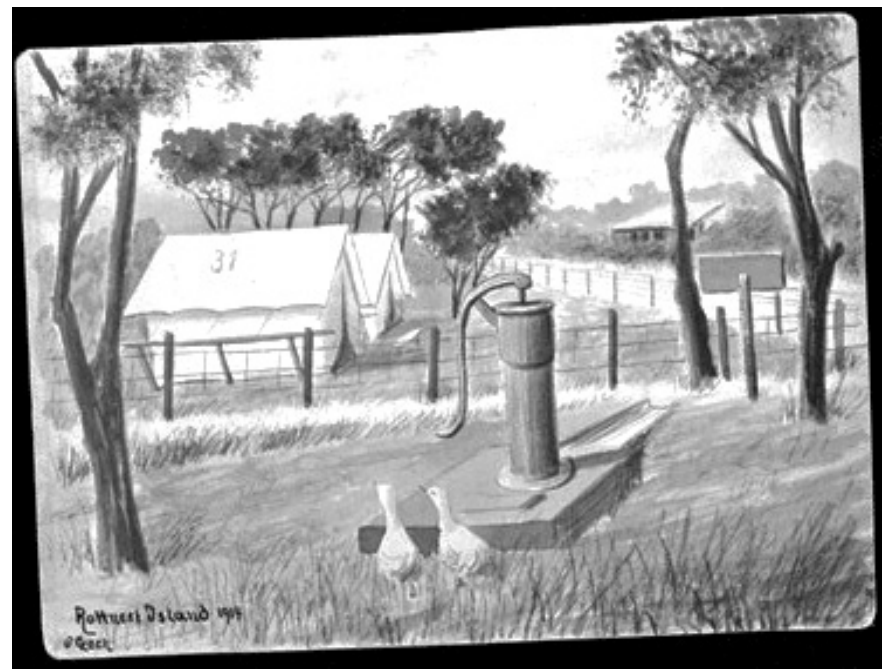

Fotografie einer Zeichnung von Erich Czech 1914, State Library of Western Australia, Picture 280256PD.

Diejenigen Internierten, die erst nach Weihnachten 1914 auf die Insel überführt wurden, waren größtenteils Westaustralier deutscher oder österreichischer Herkunft. Sie hatten nicht mehr das Glück, bereits fertiggestellte Unterkünfte vorzufinden. Einer der australischen Wachhabenden, Rupert Secombe, gab später an, dass den meisten Gefangenen einfach das nötige Material übergeben wurde und sie sich die Zelte selbst aufbauen mussten (28). Einer dieser "Nachzügler" unter den Internierten erinnert sich daran, dass Mitte 1915 eine große Zahl von Österreichern ankam, die den Deutschen bald zahlenmäßig überlegen waren. Darunter gab es viele Kroaten aus Dalmatien, die "in Zelten der australischen Armee oder gar in Freizeitzelten, die in Perth bestellt wurden, untergebracht wurden" (Splivalo 67). Wie Stedmans fiktionaler Internierter war auch Anthony Splivalo gerade 16 Jahre alt, und erst wenige Jahre zuvor aus Österreich-Ungarn nach Kalgoorlie gekommen, wo sein älterer Bruder unlängst Fuß gefasst hatte. Splivalo besuchte in diesem Goldgräberstädtchen zuerst die Schule und arbeitete dann als Büroangestellter. Obschon er sich gut integriert hatte und perfekt Englisch sprach, wurde auch er 1915 interniert, als die ortsansässigen Minenarbeiter sich der konkurrierenden Arbeitnehmerschaft entledigen wollten und 
in großer Zahl Österreicher und Angehörige der Balkannationen als Feinde isolierten und nach Rottnest Island verbannten. Dies geschah, wenngleich viele von ihnen dem Krieg und den Aggressoren vollkommen unbeteiligt gegenüberstanden. In seiner Autobiographie reflektierte Splivalo die Ereignisse viele Jahre später mit einem sehr verständnisvollen Blick. Er war eindeutig einer der Internierten, die damals wie später das Beste aus ihrer Situation machten. An sie erinnerte sich einer der Wächter wohlwollend:

Sie bauten sich sogar eine Art Kursaal, in dem abends Tanz und Musik stattfand, denn sie hatten eine richtig gute Musikkapelle. Sie bekamen Musikinstrumente und gaben Konzerte und so weiter. (Secombe 28)

Einige der Wachmänner und Sanitäter, beispielsweise Captain Maggs Randall und Gefreiter John Secombe, wurden oft zu diesen Veranstaltungen eingeladen und verschafften sich Zugang zu dem eingezäunten Gelände, indem sie sich als diensthabende Angehörige des Australian Medical Corps ausgaben. Es gab anscheinend viele Kontakte zwischen Wärtern, Wachmännern und Gefangenen. Einer dieser Wachmänner, Jack O'Donoghue, erzählte, dass zwei oder drei der Wärter einen großen Bottich besaßen, in dem sie Bier brauten, für das sie auf der Insel angebaute Gerste verwendeten sowie Hefe, die sie aus Kartoffeln und Gerste selbst hergestellt hatten (9). Auch die Gefangenen auf der anderen Seite waren damit beschäftigt, sich heimlich eine Lager-Brauerei einzurichten.

Die von einem weiteren deutschen Internierten, Otto Ferdinand Dreissig, gezeichnete Karte (siehe unten) zeigt deutlich, dass sich eine Art Schicksalsgemeinschaft gebildet hatte. Die Internierten versuchten, eine Dorfatmosphäre zu schaffen und selbst für die wichtigsten Dienstleistungen zu sorgen. Ein Zelt wird auf der Karte als Brauerei bezeichnet, andere wurden mit Bäckerei, Metzgerei, Krankenhaus, Wäscherei, Laden und Barbier beschriftet. Die Wasserklosetts markierte Dreissig mit roter Farbe, genauso wie das Gefängnis, den Strandpavillon und den Kursaal, der Monte Carlo getauft wurde.

Der 24-jährige Matrose Dreissig war Rottnest-Gefangener Nr. 63. Wie viele seiner Mitgefangenen war auch er Reservesoldat der Marine (NAA: PP14/2; 753016). Dreissig war schon einige Zeit in Australien heimisch und auf dem Dampfer Western Australia tätig gewesen, als er am 14. August 1914 in einem Hafen im Nordwesten von Australien, in Wyndham, festgenommen und als Passagier Zweiter 
Klasse nach Fremantle verfrachtet worden war (NAA: PP14/2, $\mathrm{PF} / 110$; 752065). Wie Lehmann und Czech gehörte er zu der ersten Gruppe von Internierten, die nach Rottnest Island gebracht wurden. Dreissig kam aber nicht wie der privilegierte Lehmann im Hostel unter, das in der linken oberen Ecke der Karte zu erkennen ist. Er kannte sich als einfacher Matrose offensichtlich gut in dem unablässig wachsenden Zeltdorf aus, das sich auf einem fünf Hektar großen Gebiet in der nördlichen Thomson-Bucht ausbreitete. Auf seiner relativ unüblich nach Westen ausgerichteten Karte erkennt man 80 ordentlich aufgereihte Zelte aus Planen, die sich zwischen der Thomson-Bay-Siedlung im Süden, den Hügeln mit dahinterliegendem Sportplatz im Norden, den Sandstränden der Thomson-Bucht im Osten und einem Zaun im Westen erstrecken. Wenn man bedenkt, dass in dem Hostel etwa 40 Offiziere wohnten und in jedem Zelt sechs bis acht Männer untergebracht waren, dann muss die Zahl der Lagerinsassen zur Entstehungszeit der Karte, im Mai 1915, ungefähr 600 betragen haben.

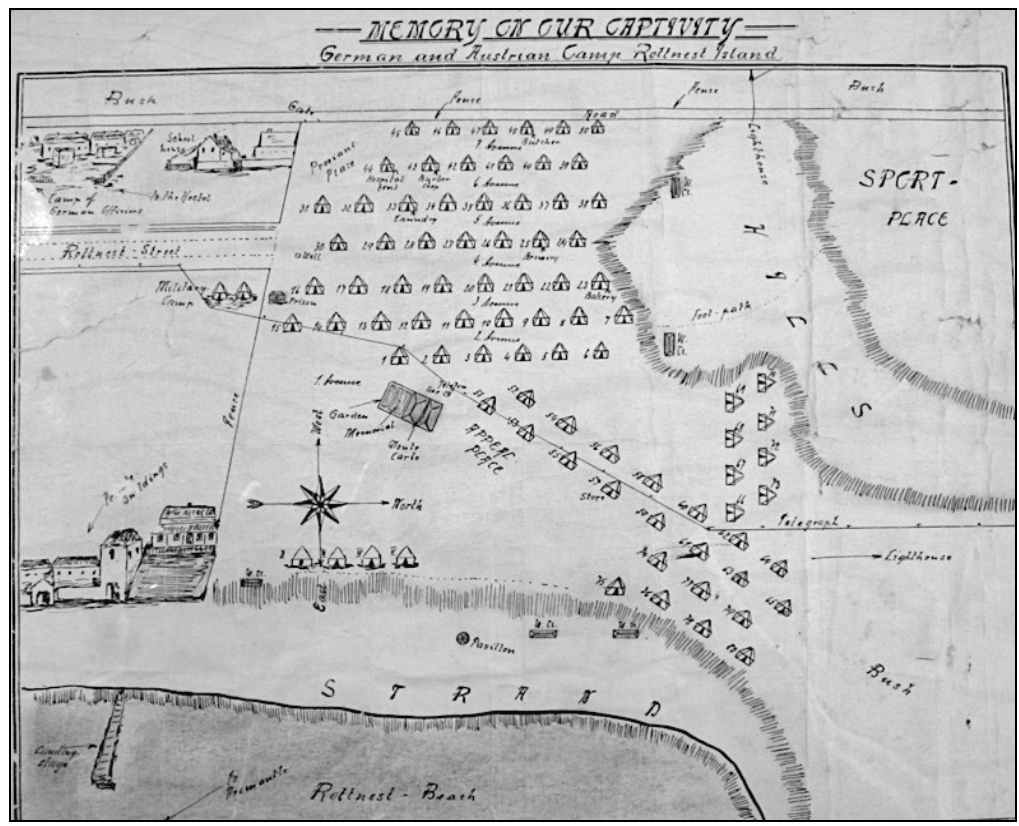

Otto Ferdinand Dreissigs Karte des Lagers von 1915, State Library of Western Australia, Map Collection, Map B/12/33. 
Die Internierten durften außerhalb des Lagers schwimmen und angeln oder im Laden der Siedlung einkaufen, eine Freiheit, die schon allein deshalb notwendig war, weil sich die Gefangenen weitgehend selbst versorgen mussten. In den Anfangszeiten des Internierungslagers wurden die abgezählten Lebensmittel, darunter auch Fleisch (Hammel) und Gemüse, vor jedem Zelt abgelegt, und die Gefangenen mussten selbst kochen. Einige Monate später wurden die Vorräte auf den Proviantplatz in der Nähe des Haupteingangs gebracht und pro Zelt wurde eine Person abgestellt, die den jeweiligen Anteil abholen sollte. Darüber hinaus gab es einen Gefangenen, "der Bäcker war" und das Lager mit Brot versorgte (O'Donoghue 9).

Anthony Splivalo erinnert sich: "Die einzige Zeitung, die uns täglich zur Verfügung gestellt wurde, war der Western Australian aus Perth, aber mein Bruder, der im Palace Hotel wohnte, sandte mir oft Ausgaben des Kalgoorlie Miners und des Western Argus" (74-75). Zweisprachige Internierte durchforsteten die verfügbaren Zeitungen nach Neuigkeiten über den Krieg oder die jeweilige Heimat und übersetzten sie für diejenigen der Lagerinsassen, die entweder Analphabeten oder des Englischen nicht mächtig waren.

Zensur war weit verbreitet und betraf nicht nur die Lagerinsassen auf Rottnest Island, sondern die gesamte Bevölkerung und vor allem die Journalisten. Daher wussten die meisten Westaustralier kaum etwas über die Situation auf der Insel. Das Leben ging für sie nach Kriegsbeginn relativ ungestört weiter. Selbst auf Rottnest Island etablierte sich erstaunlich schnell eine gewisse Alltagsroutine. Schon wenige Wochen nach Einrichtung des Lagers hatte sich eine Art "Lagerkultur" entwickelt, die hinsichtlich der sozialen Struktur und ethnischen Zugehörigkeit ein Spiegelbild der Gesellschaft außerhalb des Lagers bildete. Das heißt also, dass die Internierten im Lager sowohl nach Klassen- als auch nach Volksgruppenzugehörigkeit getrennt waren. Neben den Deutschen und den Deutsch-Österreichern gab es die Dalmatiner und Kroaten, die zusammen mit anderen Balkanvölkern die größte Volksgruppe im Lager bildeten. Es gab eindeutig soziale Unterschiede und die Rollen wurden je nach persönlichen Fähigkeiten und Qualifikationen verteilt. Diejenigen, die es sich leisten konnten, versuchten ihr Leben so angenehm wie möglich zu gestalten.

Von Anfang an gab es zwei deutlich getrennte Gruppen: die der Fremden und die der Ortsansässigen. Auf der einen Seite waren die 
Schiffsbesatzungen aus Übersee, die innerhalb Australiens keine oder nur sehr wenige Kontakte hatten, auf der anderen Seite diejenigen enemy aliens, die aus der westaustralischen Bevölkerung stammten. Viele der letzteren konnten auf die Unterstützung von Freunden zählen, einige sogar auf die Hilfe von Familienmitgliedern, vor allem diejenigen, die bereits ihr ganzes Leben in Australien verbracht hatten. Neben diesen Unterschieden in den Helfernetzwerken spielten weitere Faktoren für die Spaltung der Lagerbevölkerung in einzelne Gruppen eine Rolle. Vor allem die Schiffsbesatzungen hielten zum Missfallen vieler auch unter den Lagerbedingungen an ihren alten Rängen fest und waren klar nach sozialen Klassen bzw. beruflicher Ordnung getrennt.

Auch die einheimischen enemy aliens waren keine homogene Gruppe, sondern gehörten verschiedenen Ethnien an. Den Deutschen und Österreichern standen die große Gruppe der Dalmatiner und Kroaten, sowie einige wenige Serben, Bulgaren, Tschechen, Ungarn, Slowaken und andere Bewohner Osteuropas gegenüber (zeitweise insgesamt fast 600 Männer), die zwar offiziell als "Österreicher" galten, weil ihr Heimatland Teil der österreichisch-ungarischen Monarchie war, von ihrer inneren Überzeugung her der österreichischen Sache aber alles andere als freundlich gesinnt waren. Je nachdem, wo ihre Wurzeln in der Donaumonarchie lagen, taten sie sich mit denjenigen zusammen, die von derselben Insel, demselben Dorf, derselben Region oder derselben Volksgruppe stammten. Nach Angaben eines Internierten aus der Gruppe der Dalmatiner hatten viele von innen vorher in denselben Berufen gearbeitet, als Minenarbeiter oder Holzfäller (Splivalo 76). Die meisten Angehörigen der Balkanvölker kamen von den australischen Goldfeldern, wo der Krieg bisweilen ein willkommener Anlass war, Konkurrenz auf dem Arbeitsmarkt loszuwerden (Fischer 1988).

Die Stimmung unter den deutschen Schiffsbesatzungen war anfangs gut, waren sie doch vom unmittelbar bevorstehenden Sieg und von ihrer Rückkehr nach Deutschland noch vor Weihnachten überzeugt. Selbstbewusst trugen sie ihren Glauben an eine militärische und völkische Überlegenheit zur Schau. Als einige der deutschen Gefangenen am 2. September 1914 den Sedanstag feierten - die Erinnerung an die entscheidende Schlacht des deutsch-französischen Krieges, bei der die Preußen 1870 den französischen Kaiser Napoléon III und große Teile seiner Truppen gefangen setzten - sangen die Ma- 
trosen laut "Die Wacht am Rhein" und "Deutschland, Deutschland über alles". Als der zuständige Wachmann fragte, was los sei, antworteten sie nur: "Da musst du die Franzosen fragen!" (Penck 85). Der Lagerkommandant Major E. Summerhayes erinnerte sich 15 Jahre nach den Ereignissen, dass die Mehrheit der Deutschen im Lager kriegstauglich war.

Wären sie nach Deutschland zurückgekehrt, wären sie rekrutiert worden. Sie hatten es hier so gut getroffen, dass mehrere mir erzählten, dass sie Gott dankten, dass sie nicht nach Deutschland zurückkonnten. (7)

Viele der gefangenen Deutschen hatten nämlich einen militärischen Grad und wären auf jeden Fall eingezogen worden, hätten sie sich bei Kriegsbeginn in Deutschland befunden. Zwischen ihnen und ihren Aufsehern kam es gleichwohl zu angespannten Situationen, da "einige unter den deutschen Offizieren glaubten, ihnen gehöre die Welt" (Summerhayes 7). Die Nachricht vom Ende des Kreuzers Emden und der Anblick ihrer Schiffe, die nun ohne sie ausliefen, wird einige ihrer Hoffnungen zerstört haben. "Anfang September 1914 wurden die Dampfer Greifswald, Neumünster und Thüringen von ihrem Ankerplatz am südlichen Kai zur gegenüberliegenden Hafenseite gebracht" ("Port paragraphs" 15). Kurz danach wurden die konfiszierten Schiffe umbenannt und von der Regierung des Commonwealth für den Kriegsdienst genutzt. Die Greifswald fuhr am 16. Oktober 1914 an Rottnest Island vorbei, die Thüringen am 21. Oktober 1914 und die Neumünster am 30. Oktober 1914, alle in Richtung Osten (Signal Station Log Book 11).

Der Wunsch ihrer früheren Besatzungen, selbst in See zu stechen, mag ihren Ausdruck in der Beschäftigung mit Modellschiffbau gefunden haben. Im Gegensatz zu anderen Lagern (z.B. Holsworthy und Trial Bay), wo die Veröffentlichung von Zeitschriften und Konzertprogrammen vom intellektuellen Leben der Internierten zeugen, haben die Insassen von Rottnest Island kein derartiges Quellenmaterial hinterlassen. Die Insassen verfolgten offensichtlich andere Ziele, und das Lagerleben war eher von Naturerlebnissen und Freiluftaktivitäten geprägt. "Eine Art Robinson-Crusoe-Erfahrung muss Teil ihres Alltags gewesen sein, schließlich war die Insel ein Paradies für Jäger und Angler" ("Rottnest Island" 2). Als einzige Zeugnisse von Internierten sind uns die Fotos Lehmanns, die Zeichnungen Czechs und die Karte Dreissigs überliefert. Nicht einmal die Modellschiffe, welche 
die deutschen Seeleute bauten und auf den Salzseen der Insel fahren ließen, sind erhalten geblieben.

\section{Der Abschied von Westaustralien}

Als das Internierungslager von Rottnest Island bereits 15 Monate nach Kriegsbeginn wieder geschlossen wurde und alle Internierten zu anderen Lagern im Osten des Kontinents transferiert wurden, waren die Gepäckbestimmungen nicht sehr großzügig, sodass viele Dinge zurückgelassen werden mussten. Die Hauptgründe für die Schließung waren die Überbelegung und dadurch hervorgerufene Versorgungsprobleme. Die Zahl der Internierten war bis dahin stetig angewachsen: Im März 1915 gab es 412 Lagerbewohner, im Mai desselben Jahres war die Zahl auf 628 Internierte angestiegen, Ende Juli auf 887 und Ende August wurden sogar 1100 Personen auf Rottnest Island festgehalten (NAA, CRS 172, 1917/4052, 47835). Die Infrastruktur auf Rottnest Island war für einen solchen Zuwachs der Bevölkerung nicht ausgerichtet, und da jedes einzelne Ausrüstungsteil mit dem Schiff herangebracht werden musste, konnte eine lückenlose Versorgung nur schwer erzielt werden. Deshalb wurde nach anderen Orten für ein Internierungslager gesucht, und man beschloss, die etwa 1000 Männer nach New South Wales zu verlegen.
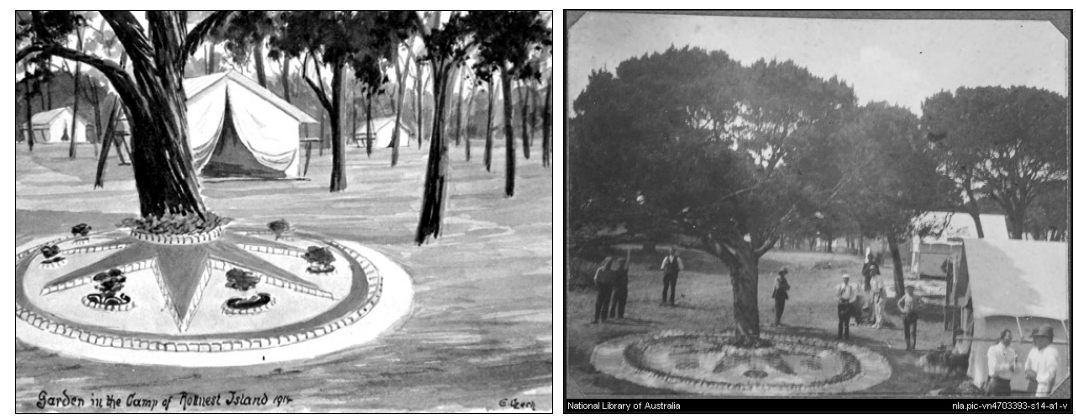

Zeichnung von Erich Czech 1914; State Library of Western Australia: no. 004543D; Fotografie von Karl Lehmann, National Library of Australia, Bib ID 4703393.

Der aus Dalmatien stammende Gefangene Splivalo gab folgende Erinnerung zu Protokoll: 
Ende 1915 überschlugen sich die Gerüchte. Wir hörten, dass die Behörden beschlossen hatten, das Lager aufzulösen und uns in ein großes Internierungslager in der Nähe von Sydney in New South Wales zu verlegen. Die Aussicht, Rottnest Island verlassen zu müssen, traf uns hart. Wir waren zwar offiziell Kriegsgefangene und lebten hinter einem Zaun, hatten auf Rottnest aber sonst keinerlei belastende Einschränkungen zu erdulden. Wir wurden nicht schlecht behandelt, und jeder, der diese zauberhafte Insel nach Herzenslust erkunden wollte, bekam dafür einen Passierschein. Wir fühlten uns inzwischen heimisch hier und hatten uns alle ausnahmslos in die Insel verliebt. Jeden Morgen konnten wir es kaum erwarten, die Insel zu durchforsten, barfuß über die silberglänzenden Strände zu wandern und die frische, saubere Luft einzuatmen. (80)

Erst wenige Tage vor dem geplanten Transfer wurden Pläne bekannt gegeben, wonach die auf Rottnest Island internierten Deutschen und Österreicher an die Ostküste Australiens verlegt und die Insel wieder den einheimischen Weihnachtsurlaubern zur Verfügung gestellt werden sollte. Diese Nachricht kam sowohl für die Lagerinsassen als auch für ihre Verwandten und die breite Öffentlichkeit mehr als überraschend. Um Unruhe und Proteste zu vermeiden, hatten die Behörden ihre Pläne so lange wie möglich geheim gehalten; auch die Evakuierung der Insassen und ihr Transfer auf einen Ozeandampfer wurde "ohne unnötiges Gedöns" ausgeführt ("Restoration of Rottnest" 1).

Am 22. November 1915 legte das Linienschiff SS Demosthenes abends um 21 Uhr ab (Splivalo 82). Die meisten der Insassen von Rottnest Island wurden nach Sydney gebracht (Mennicken-Coley 64). Czech und Dreissig kamen in das Lager von Holsworthy, während einige Privilegierte, darunter Lehmann, nach Trial Bay überführt wurden. Erst 1919 sollten diese Männer wieder in See stechen, als sie auf einem der zwischen 1919 und 1920 speziell dafür bereitgestellten Schiffe zurück nach Deutschland geschickt wurden (Scott 137). Zusammen mit den vielen Seemännern, die auf der Durchreise gefangengenommen worden waren, wurden auch viele tausend Deutsche und Österreicher repatriiert, die teilweise schon sehr lange in Australien lebten. Es machte keinen Unterschied, ob man eingebürgert war (naturalisiert) oder gar sein Leben lang in Australien wohnte. Das Kriegsende bot die Möglichkeit, das Land "britischer" zu machen, und viele Familien deutschen und österreichischen Ursprungs verließen nun, teils gezwungenermaßen, teils freiwillig, das Land. 
Bevor die Internierten abgeschoben wurden, fotografierte man die Männer, wohl auch, um sie bei dem unerlaubten Versuch der Wiedereinreise leichter identifizieren zu können. Lehmann wurde auf der Kursk repatriiert, die Sydney am 29. März 1919 verließ, Erich Czech und Otto Dreissig wurden sechs Wochen später, am 9. Juli 1919 auf der Tras-os-Montes deportiert. Beide Schiffe fuhren auf ihrem Weg nach Europa nicht über Fremantle, sondern liefen vor der Überfahrt des Indischen Ozeans nur noch den Hafen von Albany an, sodass die Männer keinen Blick auf die mittlerweile wieder für den Tourismus freigegebene Insel Rottnest werfen konnten.

Anthony Splivalo war, wie in Stedmans Roman, einer der wenigen "Österreicher", die in Westaustralien bleiben durften. Doch auch er entzog sich der feindseligen Atmosphäre dieser Jahre und emigrierte in die USA. Dank Splivalos Autobiographie, Lehmanns Fotos, Dreissigs Landkarte und Czechs Zeichnungen können wir uns heute immer noch ein gutes Bild von dem Moment in der Geschichte Australiens machen, als sich auch hier unter dem Eindruck der Weltpolitik die unterschiedlichen Einwanderergruppen voneinander distanzierten und untereinander alte Ressentiments neu aufbrachen.

\section{Bibliografie}

\section{Bildmaterial}

Czech, Erich, 1914/15. Six Rottnest Island Paintings from 1914 and 15, Image Catalogue, State Library of Western Australia.

Dreissig, Otto Ferdinand, 1914. "Map of the camp from 1914", State Library of Western Australia, Map Collection, Map B/12/33.

Lehmann, Karl, 1914-1918. German Concentration Camp Australia, Album of Internment Camps on Rottnest Island, Western Australia and at Trial Bay and Holsworthy, New South Wales, 1914-1918, http://nla.gov.au/nla.pic-vn4703393.

\section{Zeitgenössische Sekundärquellen}

Commonwealth of Australia Gazette, 1914. No. 56, Thursday, 13 August. 
Gossip, 1914. "On Active Service. Goldfielders in the Camp", Westralian Worker. Official Organ of the West Australian Labor Party, 13 November: 3.

Minute Paper by the Attorney General's Department, dated 15/02/ 1916. PP14/2, 30233301, NAA (Perth).

NAA. National Archives of Australia, various files on Rottnest Island's internment camp.

O'Donoghue, Jack, 1987. "Oral history interview, 6 August 1987", transcribed by Geraldine Varang, Murdoch University History Club, OH1987.1, Tape 2.

Penck, Dr Albrecht, 1915. Von England festgehalten. Meine Erlebnisse während des Krieges im britischen Reich. Stuttgart: Verlag von I. Engelshorn.

Scott, Ernest, 1941. Australia During the War. The Official History of Australia in the War of 1914-1918, vol. XI, Sydney: Angus and Robertson Ltd.

Secombe, John Rupert, 1977. "Oral history recording. 1977", $\mathrm{OH} 253$, State Library (WA).

Senator Pearce, 1915. "Statement in Parliament", Argus, 28 May.

Signal Station Log Book (Rottnest Island), 1913-1915, State Record Office, 3493, Series 118, RIL11.

Summerhayes,E., 1930. "Prisoners of War. Rottnest Reminiscences", The West Australian, Thursday, 27 February: 7.

The Advertiser (Adelaide, SA), 1914. "The censors at work. Cable messages delayed", 7 August: 15.

The Register (Adelaide), 1915. "Pleasure seekers and prisoners", Saturday, 27 November: 10.

The Sunday Times (Perth), 1914. "Port paragraphs", Sunday, 6 September: 15.

The Sunday Times (Perth), 1915. "Restoration of Rottnest. Alien Enemy Removed", Sunday, 28 November: 1.

The Western Mail (Perth), 1914. "Captured off Fremantle", Friday, 21 August: 14.

The Western Mail (Perth), 1937. "Rottnest Island", Thursday, 18 November: 2.

The Westralian Worker, 1914. "Notes from the Sea", 14 August: 10. 


\section{Heutige Forschungsliteratur}

Fischer, Gerhard, 1988. "Enemy labour: Industrial Unrest and the Internment of Yugoslavs in Western Australia during World War I", Australian Journal of Politics and History, 34, 1: 1-15.

Fischer, Gerhard, 1989. Enemy Aliens. Internment and the Homefront Experience in Australia 1914-1920. St Lucia: University of Queensland Press.

Helmi, Nadine, and Gerhard Fischer, et al., 2011. The Enemy at Home. German Internees in World War I Australia. Sydney: UNSW Press.

Mennicken-Coley, Mary, 1993. The Germans in Western Australia. Innovators, Immigrants, Internees, Mount Lawley: Cross Print, ECU.

Simons, John, 1999. Prisoner in Arcady: German Mariners in Berrima 1915-1919. Berrima: Berrima District Historical \& Family History Society.

Splivalo, Anthony, 1982. The Home Fires, Fremantle: Fremantle Arts Centre Press.

Stedman, M.L., 2012. The Light Between Oceans. Novel. Sydney: Random House / Vintage Books. 